\title{
Hardware Considerations to Optimize Zernike Phase Contrast TEM for Cryo-Tomography and Single Particle Data Acquisition
}

\author{
B.L. Armbruster*, J. Brink*, R. Danev**, T. Isabell*, M. Kawasaki*, M. Marko***, S. \\ Motoki**** and K. Nagayama** \\ *JEOL USA Inc., Peabody MA 01960 \\ **Okazaki Institute for Integrative Bioscience, National Institutes of Natural Sciences, Dept. \\ Physiological Sciences, The Graduate University of Advanced Studies, Higashiyama 5-1, \\ Myodaijicho, Okazaki 444-8787 Aichi, Japan \\ ***Wadsworth Center, New York State Department of Health, Albany, NY 12201 \\ ****JEOL Ltd., 1-2 Musashino 3-chome, Akishima Tokyo 196-8558, Japan
}

Phase contrast TEM imaging with Zernike phase plates has been successfully applied to produce images of vitreously frozen specimens, including cryosections, whole cells and single particles, with optimum contrast at or near true focus [1-7]. Several column designs from 120$400 \mathrm{kV}$ have been modified to include a phase plate holder inserted in the back focal plane: Hosokawa et al [8] described a 120kV design incorporating an additional lens doublet and heated phase plate holder, Motoki et al [9] presented high resolution images of amorphous germanium and graphite taken on a $200 \mathrm{kV}$ platform with the phase plate positioned at a conjugate back focal plane, the $3.5 \mathrm{~nm}$ structure of rat TRPV4 cation channel was reconstructed from images taken in a $300 \mathrm{kV}$ phase contrast TEM equipped with a liquid helium stage [10], and critical experiments to understand phase contrast optics and improve the quality of phase plates continue [5].

As discussed in Danev et al [5], Marko et al [11] and Armbruster et al [12], the optimal design for phase contrast single particle imaging and cryo-electron tomography requires a thorough review of all optical elements to increase image contrast and automation capabilities to maximize ease of use. Features required in a dedicated column configuration have been incorporated in the JEM-2200FS (Fig. 1a). The JEM-2200FS 200kV TEM features a fieldemission gun, a heated phase plate holder, an objective focal length of $5 \mathrm{~mm}$ or larger to accommodate the phase plate holder, plus a room-temperature aperture between the cryospecimen and phase plate holder to serve as a heat shield. The phase plate holder, shown in Figs. $1 \mathrm{~b}$ and $1 \mathrm{c}$, includes $\mathrm{X}$ and $\mathrm{Y}$ piezo drives for precision positioning, as well as continuous heating of the phase plate up to $200^{\circ} \mathrm{C}$ to minimize charging and contamination of the carbon film. The phase plate holder accepts multiple phase plate disks, and each disk can have 25 phase plates [4]. The small size of the central hole of the carbon phase plate (Fig. 1d), typically less than $1 \mu \mathrm{m}$ in diameter, requires precise hardware and software control to center parallel illumination in all low dose modes and tilt angles. Zero-loss energy filtering, provided by the in-column Omega filter, doubles the signal-to-noise ratio [13] and is essential for attenuating inelastic scattering when imaging thick samples such as whole cells or vitreous cryosections.

\section{References}

[1] K. Nagayama and R. Danev, Phil. Trans. Roy. Soc. B (2008) 2153.

[2] M. Yamaguchi et al., J. Struct. Biol. 162 (2008) 271.

[3] R. Danev and K. Nagayama, J. Struct. Biol. 161 (2008) 211.

[4] K. Nagayama, Eur. Biophys. J. 37 (2008) 345.

[5] R. Danev et al., Ultramicroscopy 109 (2009) 312. 
[6] Y. Fukuda et al., J. Struct. Biol. 168 (2009) 476.

[7] M. Marko et al., Proc. 16th Int. Congress on Microscopy 1 (2006) 100.

[8] F. Hosokawa et al., J. Elec. Microsc. 54 (2005) 317.

[9] S. Motoki et al., Microsc. Microanal. 11 (Suppl 2) (2005) 708.

[10] H. Shigematsu et al., J. Biol. Chem. E-pub Dec. 31, 2009.

[11] M. Marko et al., Microsc. Microanal. 13 (Suppl 2) (2007) 1212CD.

[12] B.L. Armbruster et al., Microsc. Microanal. 14 (Suppl 2) (2008) 1072.

[13] R. Grimm et al., J. Microsc. 190 (1998) 339.
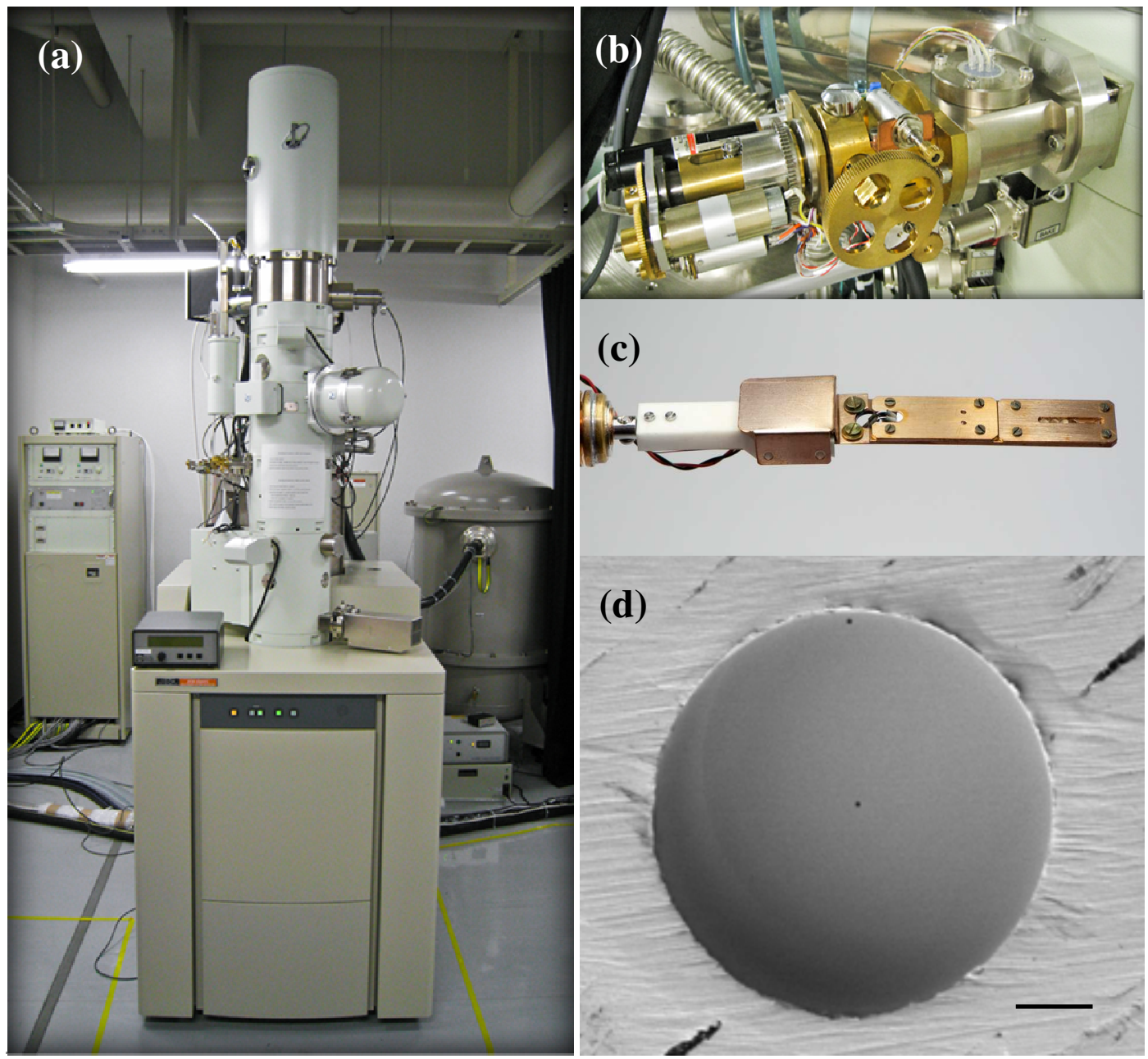

Figure 1. Phase contrast TEM at Okazaki Institute for Integrative Bioscience. (a) The JEM-2200FS includes a dedicated phase plate cryopolepiece and phase plate holder. (b) Phase plate holder motor drive assembly with piezo fine control, cover removed. (c) Tip of holder assembly for heated phase plate incorporates ceramic insulators, heaters and space for multiple phase plate disks. (d) SEM image of a 50 $\mu \mathrm{m}$ diameter carbon phase plate with $1 \mu \mathrm{m}$ central hole, scale bar $=10 \mu \mathrm{m}$. 